\title{
Análisis de un tipo de intervención terapéutica para niños y adolescentes con trastornos de comportamiento
}

RESUMEN: En este trabajo se analizan los resultados obtenidos con una intervención sistémica sobre una muestra de 16 preadolescentes y adolescentes con diversos trastornos de comportamiento.

PALABRAS CLAVE: Trastornos de comportamiento, Terapia Familiar, Sistémica, Niños, Adolescentes
SUMMARY: In this work, the authors analyze the outcomes that they have been obtained accomplishing a Systemic Family Therapy on a sample of 16 pre-adolescent and adolescent with behavior disorders. KEY WORDS: Behavior Disorder, Family Therapy, Systemic, Child, Adolescent

\section{1.- Introducción}

El consultorio familiar donde trabajamos los autores es de carácter privado y recibe demandas de casos que en una gran proporción están relacionados con diferentes trastornos de comportamiento de niños y adolescentes. Los pacientes habitualmente llegan derivados por otros profesionales tanto del trabajo social como clínicos de psiquiatría y psicología. Antes de proseguir queremos aclarar que hemos empleado el concepto de Trastorno de Comportamiento para referimos a un grupo quizás heterogéneo de síntomas, que tienen como elemento común que la demanda de tratamiento es realizada por algún familiar, generalmente los padres, que se presentan muy angustiados y a veces deprimidos ante la presencia del problema.

Como decimos, los «síntomas» comportamentales que hemos tratado han sido muy variados, yendo desde leves trastornos, consistentes básicamente en conductas de desafío o provocación hacia los padres, hasta situaciones más dramáticas de agresión hacia ellos. Fracaso escolar, consumo de sustancias prohibidas o de alcohol, robos domésticos, amenazas a los padres, fugas del domicilio, crisis histéricas, pataletas con y sin agresiones hacia objetos, problemas de identificación sexual, hurtos, retraimiento social, inhibición, depresión, angustia, anorexia, desobediencia, etc. se han presentado en diferentes combinaciones en cada uno de los casos. Pero como ya hemos comentado, quizás el elemento común de entrada haya sido que la necesidad de consultar o pedir ayuda estaba ubicada en la familia.

Este tipo de casos nos ha llevado a reflexionar sobre tres elementos que están en interacción. 1) La adolescencia en sí, desde la perspectiva del individuo, como etapa de paso del crecimiento y al mismo tiempo como periodo especialmente difícil de la vida. 2) El contexto familiar donde aparece el problema. 3) Y por último la intervención terapéutica que hemos elegido para trabajar los casos y que nos va a llevar a analizarla desde el plano de los resultados obtenidos: el abordaje estructural. 
Sin lugar a dudas, nuestra formación como terapeutas relacionales ha determinado que seleccionemos un tipo de intervención familiar sistémica para estos casos, así como que entendamos la función relacional del síntoma en el contexto familiar. La hipótesis de partida para ello es que los trastornos de comportamiento del adolescente son fruto de dificultades familiares de diferente índole, pero generalmente estos problemas están centrados en la organización familiar, en su estructura. Por tanto hemos empleado un tipo de intervención estructural, es decir, dirigida a recomponer una estructura familiar funcional. El interés de este trabajo es discutir si esta hipótesis es generalizable a todos los casos y de no ser así, qué indicadores relacionados con el tipo de trastorno o con el funcionamiento familiar nos podrían ayudar a seleccionar otro tipo de intervención más fructífera para ellos.

\section{2.- Algunas consideraciones sobre la adolescencia}

Desde nuestro punto de vista hay dos ejes sobre los que se asienta la etapa que llamamos adolescencia: a) El proceso de crecimiento (en todos los órdenes, biológicos, psicológicos y sociales) hacia la edad adulta y b) la vinculación familiar.

Resulta obvio que a nivel madurativo, la adolescencia es un periodo de tránsito donde el niño empieza a transformarse biológicamente en un joven adulto, al mismo tiempo que psicológicamente inicia una fase de diferenciación y de apropiación de elementos de identidad. Estos dos fenómenos tienen una traducción social en cuanto facilitan una conexión más íntima con el mundo exterior a través del establecimiento de las relaciones amistosas y amorosas.

En un segundo plano, el adolescente puede crecer en la medida en que se mantiene vinculado a su familia de origen y el grupo familiar permite su diferenciación. Desde esta perspectiva, el punto de partida de la adolescencia es la infancia tardía, donde todavía hay un nivel máximo de dependencia emocional y organizativa del individuo con respecto a la familia, y discurre hacia otro punto en el que el joven adulto es capaz por sí mismo de autoorganizarse y de establecer vínculos emocionales sólidos con personas externas al núcleo familiar. El lapso de tiempo en el que esto ocurre es variable aunque en nuestros días y en nuestro medio tiende a prolongarse.

No obstante, algunos autores cuestionan la adolescencia como una etapa de la vida. M. Rutter [citado por Fishman (1)] dice: «La adolescencia es reconocida y tratada como una etapa distintiva del desarrollo debido a que la coincidencia de una educación más extensa y una maduración sexual temprana han dado lugar a una fase prolongada de madurez física asociada con una dependencia económica y psicosocial; a que muchas de las teorías psicológicas ampliamente aceptadas especifican que la adolescencia debería ser diferente; a que los intereses comerciales exigen una cultura de los jóvenes y a que los colegios y universidades determinan que grandes cantidades de jóvenes se mantengan juntos en un grupo social segregado por edad (Rutter, 1980). El mismo Fishman (1), empero, hace hincapié en que la adolescencia debe contemplarse como una transformación social más que biológica. 
ORIGINALES Y REVISIONES

Sea considerada la adolescencia una etapa del ciclo vital o fruto de una construcción social en un momento histórico dado, la cuestión es que los clínicos nos encontramos con un grupo de personas jóvenes que plantean una serie de problemas sociofamiliares que dificultan tanto el crecimiento individual como el familiar. Y que estas personas y sus familias se encuentran en un estadio evolutivo de su etapa de crecimiento que tiene que ver con el proceso de desvinculación.

Haley (2) comenta que «la época de mayores cambios en cualquier organización sobreviene cuando alguien se incorpora a ella o la abandona». Por definición, la preparación del joven para ser independiente, para «salir» de la familia organizativa y emocionalmente, conllevará una serie de conflictos en su ámbito familiar, originados por la necesidad de modificar las pautas y normas de relación vigentes hasta entonces, por otras nuevas que permitan, en condiciones normales, una mayor adaptación de la familia a las nuevas capacidades del individuo y de éste, por tanto, a la posibilidad de integrarse plenamente en nuevos contextos relacionales.

M. Knobel (3) dice que la adolescencia es "una situación que obliga al individuo a reformularse los conceptos que tiene acerca de sí mismo y que lo lleva a abandonar su autoimagen infantil y a proyectarse en el futuro de su adultez». Y añade que las manifestaciones sintomáticas del adolescente forman una entidad semipatológica que ha denominado el Síndrome Normal de la Adolescencia. De todos lo fenómenos que señala este autor que se producen en la adolescencia ${ }^{1}$, a nosotros nos interesa, desde la perspectiva en la que trabajamos, poner énfasis en algunos de ellos, dado que nos serán útiles para el análisis de los resultados de nuestro trabajo. La necesidad de construcción de la propia identidad nos parece el más relevante por cuanto implica cambios en el propio individuo que estarán condicionados por la adaptación familiar a los mismos. Para Sorenson [citado por Knobel (3)], la identidad es la "creación de un sentimiento interno de mismidad y continuidad, una unidad de la personalidad sentida por el individuo y reconocida por otro, que es el saber quien soy". Dice el vocabulario de Terapia Familiar (4) que la identidad es "el sentimiento de ser alguien quien, a pesar de los cambios experimentados por las circunstancias, los estados físicos y las relaciones, permanece constante; en otras palabras, manifiesta continuidad y coherencia". Comentaremos seguidamente alguno de los elementos que nos parecen más relevantes en la construcción de la identidad: la apropiación de los deseos, la aparición de la intimidad y la transgresión de las normas.

$\mathrm{Al}$ inicio de la adolescencia se empieza a dar el paso de ser lo que uno es por razón de ser hijo de sus padres a tratar de saber quien es uno en sí mismo como persona distinta de los padres [Waddell, M. (4)]. En la medida en que la afirmación en los propios deseos contribuye a la construcción de la identidad, y a la diferenciación con respecto a los padres, observamos como en la historia del deseo se operan una serie de cambios: 1) En primer lugar, va pasando en la primera infancia de la inmediatez en su cobertura, a la mediatez (con lo cual comienza el aprendizaje sobre la tolerancia a la frustración). 2) En un segundo momento el niño, cuyos deseos son influidos por los de los padres (consciente o inconscientemente) va a pasar en la adolescencia a elegir sus propios deseos, a ser pro-

' Búsqueda de identidad, tendencia grupal, necesidad de intelectualizar y de fantasear, crisis religiosas, desubicación temporal, evolución sexual, actitud reivindicativa, contradictoriedad, separación progresiva de los padres y fluctuaciones del estado de ánimo. 
tagonista de los mismos. De desear algo porque está en la expectativa de los padres a desear algo porque lo quiere él.

En uno de los casos que tratamos, el paciente, un joven de 18 años, debuta con un cuadro clínico muy aparatoso tras el consumo de un cigarrillo de cannabis: una crisis de pánico con una fuerte reacción de despersonalización. Una vez pasada la crisis, el paciente manifiesta una sensación de vacio, de no ser nadie y acepta una terapia individual con el objetivo de conocerse, de saber quien es él. Hasta ese momento, el joven desarrolla una vida un tanto inconformista: líder de su grupo de pares, con ideas revolucionarias $e$ utópicas sobre la solidaridad, la injusticia, las causa s perdidas, etc. se había granjeado la enemistad de sus profesores de instituto y había fracasado en su incorporación a la universidad. Siendo fiel a su ideario social se había buscado empleo como jornalero en el campo. Su padre era aparejador y su madre profesora de instituto. Mantenía una relación confrontativa con el padre y de alianza y apoyo por parte de la madre. En una de las entrevistas con los padres a solas, la madre nos confesó su admiración por esas cualidades inconformistas de su hijo. De hecho ella militaba en movimientos sociales solidarios aunque sin el nivel de compromiso personal que sostenía su hijo. En su admiración había un refuerzo de la conducta desadaptativa del chico que a nuestro parecer había dedicado su adolescencia a gratificar esos deseos de la madre. En el momento de la crisis, entendimos que lo que se había puesto de manifiesto era un problema en el nivel de los deseos: ser solidario porque uno quiere o serlo porque es lo que los padres esperan de uno. Y en la medida en que el deseo propio nos da identidad, resultó que el chico, al tomar conciencia de su situación, se quedó sin saber qué deseaba él, y por tanto, con dificultades para autoreconocerse.

Un segundo factor que nos interesa analizar es el del establecimiento de fronteras nítidas entre el mundo psíquico del joven y el universo familiar. La individuación es un proceso por el cual el sujeto llega a establecer límites psíquicos frente a los miembros de la familia. Según Cancrini (6) hay que distinguir el momento de la primera individuación que sucedería al término de la simbiosis psicológica con la madre, de la segunda individuación marcada por el comienzo de las relaciones con el exterior y que coincide aproximadamente en el tiempo con el inicio de la escolaridad. En la infancia, por tanto, los pensamientos y los sentimientos van pasando de una situación en la cual son comunicados conforme fluyen, y por ello pueden ser conocidos por otros miembros de la familia hacia otra de mayor control en su emisión, hasta el punto en que en la adolescencia se consolida la intimidad: la necesidad de reservarse para sí mismo cierto tipo de pensamientos, de fantasías, de sentimientos o actos que no pueden o deben ser conocidos o compartidos por otras personas de la familia o sólo por las que el joven quiere. Dado que en esta época coincide la eclosión de la sexualidad, la intimidad cobra una especial importancia de cara a establecer los límites del yo. El joven comienza a tener secretos que no se pueden/deben compartir con los padres, porque el compartirlos supone, precisamente no disponer de espacio propio y son una invitación a continuar anclados en la etapa de la niñez.

En otro de los casos que tratamos, un chico de 16 años que presentaba una conducta de desafío hacia los padres, manifestada a través de desplantes, portazos, y amena- 
zas suicidas, el paciente, al que los padres vigilan y controlan porque temen que pueda estar fumando cigarrillos de cannabis, les confiesa abiertamente en una sesión que efectivamente durante el último verano ha estado fumando porros todos los días. Inmediatamente después de comunicar esto a los padres dice haberse arrepentido porque piensa que ahora le controlarán más, curiosamente cuando el se estaba quejando del excesivo control al que lo tenían sometido los padres. Nuevamente aqui encontramos que compartir algo que debía ser secreto forma parte de un juego relacional donde se invita explícitamente a los padres a participar: así el chico mantiene su atadura a los padres alrededor del control que el mismo provoca. Se puede quejar de que lo tratan como a un niño pequeño, siendo el mismo, con su indiscreción, el que sostiene el trato.

Un último fenómeno que nos interesa analizar desde la perspectiva de nuestro trabajo es que, fruto de esos movimientos por obtener identidad y diferenciación, aparece la necesidad de transgredir las normas familiares. El adolescente necesita tocar los límites: forma parte de un proceso de tanteo de sus capacidades (Waddell). Y tocar los límites o transgredirlos tiene una utilidad fundamental: obtener espacios cada vez más amplios para el desarrollo de las propias competencias y como consecuencia iniciar el cambio adaptativo en la familia. Pero también en la transgresión o en la oposición a las pautas y reglas familiares, el adolescente encuentra un elemento de la realidad que le devuelve que él es él: un ser diferente, protagonista, lleno de deseos propios y de voluntad. Llegar a las doce y media a casa cuando los padres le han dicho que tiene que estar a las doce forma parte de este doble juego: por un lado sentir que uno hace lo que debe porque se ve capacitado para ello y no necesita del control externo que imponen los padres, pero al mismo tiempo ayuda a estos a poder cambiar y aceptar que el joven está creciendo, aunque para ello sea preciso llegar a establecer una guerra. Las fuerzas que se oponen serán, por un lado la necesidad de los padres por mantener el statu quo, y por otra, la presión del joven para cambiar y tener mas espacio (autonomía, libertad). Si los padres son figuras de apego seguras, el adolescente tiene más facilidad para explorar su competencia y autonomía (7). En cualquier caso, para lograr una relación positiva entre los padres y el adolescente, los padres deberán ir transformando gradualmente el uso de la autoridad desde una aproximación unilateral a una relación mas cooperativa y de co-construcción (8). Es decir, que la posible guerra debe dar lugar a una negociación en la que los padres van aceptando y reconociendo las capacidades del joven. En la medida en que el adolescente conquista espacio, esto se convierte en un elemento que refuerza su identidad. Pero es justamente la sensación de conquista, es decir, que los logros son el resultado de una lucha o una negociación, lo que le permite al joven obtener identidad, y al mismo tiempo, la adaptación de los padres al nuevo cambio. Con ello lo que queremos decir es que los logros obtenidos con la anuencia de los padres sin negociar o presentar batalla, no le permitirán al joven reconocerlos como propios y por tanto no serán utiles a los fines de conseguir sentimiento de identidad. Si el chico transgrede una norma familiar y los padres lo toleran, él necesitará aumentar en calidad o cantidad la transgresión hasta sentir que les "arranca" a los padres su "conquista". Teñirse el pelo de azul o colocarse un pendiente en la adolescencia, en contra del criterio estético de la familia, supone un paso hacia la consecución de identidad. 
Si la familia acepta esta transgresión a sus normas estéticas sin presentar "batalla", estos dos hechos pierden la cualidad psicológica de servir al fin de la identificación y por tanto, serán una invitación implícita a que la transgresión adquiera una mayor intensidad.

Es normal que existan conflictos en la relación de los padres con los adolescentes, pero no lo es que dichos conflictos sean frecuentes, intensos y no se puedan resolver (9). Entendemos, por tanto, que en la adolescencia será esperable un cierto grado de conflictividad que no podrá ser connotada como disfuncional o patológica. De hecho, todas las personas pasamos por este periodo y somos capaces de superarlo exitosamente en la mayoría de los casos. Que dicha conflictividad se convierta en un problema para el que haya que solicitar ayuda dependerá de una serie de factores individuales y familiares que analizaremos a lo largo de este trabajo. Pero también hay factores de la esfera social que pueden influir en el problema. Pensemos, por ejemplo, en los mensajes contradictorios o francamente confusos que damos los profesionales cuando unos aconsejamos sobre la conveniencia de mantener los límites de forma nítida y otros mantenemos que eso puede ser contraproducente. O cuando algún estamento de la comunidad (servicios sociales, judiciales o policiales) se permite criticar la manera de ejercer la autoridad de los progenitores, que quedan desprotegidos y descalificados ante el adolescente.

\section{3.- El contexto familiar en la adolescencia}

Durante la época de la adolescencia, el sistema familiar entrará en una de sus crisis más decisivas desde el punto de vista psicopatológico. La tendencia de la familia a continuar con las mismas pautas y reglas de la infancia, ante jóvenes adultos que requieren otro tipo de trato, determinará la aparición de tensiones relacionales que se deberán ir resolviendo de forma que cambien las normas familiares. Este procesó, como ya hemos comentado, permitirá mayores espacios de autonomía para el joven (organizativa y emocional) y mayor adaptación de la familia a estos cambios, con lo que se regulará y disminuirá dicha tensión. Boszormenyi-Nagy (10) afirma que la desvinculación o separación debieran considerarse una meta importante para la salud del núcleo familiar. "Como proceso emotivo, la separación es la expresión de una fase crucial del desarrollo de la familia entera. En la medida en que cada miembro de la familia es parte importante del trasfondo dialéctico de la personalidad de los otros, perder al Otro como compañero de diálogo es una pérdida dolorosa para la configuración sujeto-objeto... El desapego de un miembro de la familia no sólo daña las relaciones directas de quien se va con cada uno de los demás miembros sino que inicia una cadena de reacciones de cambios relacionales compensatorios entre los restantes miembros del sistema familiar". Y añade este autor "la separación es un proceso bastante complicado, y para su logro exige que se hayan alcanzado de modo satisfactorio las metas de la filiación y la individuación. Sólo si mantuvo relaciones estrechas, confiadas y recíprocas con los miembros de la familia, y si esas relaciones fueron interiorizadas, la prole estará en condiciones de cortar los vínculos familiares y reemplazarlos por vínculos extrafamiliares". 
En general, las familias afrontan muchas exigencias a través de su ciclo vital. A menudo se las describe como transiciones, pasajes, tareas evolutivas de la familia y sucesos en la vida familiar. Las familias con hijos adolescentes necesitan una estructura viable para desempeñar sus tareas esenciales, una de las cuales, de suma importancia, es el apoyo a la individuación, pero atendiendo al mismo tiempo a la necesidad de proporcionar un sentimiento de pertenencia.

El subsistema parental tiene que ir modificándose a medida que el hijo va entrando en la adolescencia. Con el aumento de sus capacidades, se le deben dar más oportunidades para que tome decisiones y se controle a sí mismo. La modalidad de negociación con adolescentes es distinta a la de hijos pequeños, es necesario exigirles más responsabilidad y concederles más autoridad. En condiciones normales, los padres deberán ir adaptándose paulatinamente a estos cambios demandados por el crecimiento del niño, no sin la angustia que supone correr el riesgo de conceder confianza en que el chico afrontará adecuadamente sus nuevos desafíos sociales. Esto supone que los padres deberán aceptar y asumir un cierto grado de inseguridad, que irá cediendo conforme el chico muestre capacidad de adaptación a los nuevos requerimientos del medio. Por tanto, los padres deberán estar en condiciones de tolerar, por un lado la situación generada por las reivindicaciones del adolescente en su búsqueda de elementos de identidad, y por otro lado, soportar la inseguridad de que sabrá responder a la confianza que solicita. Sin duda, los cambios del adolescente deben ser correspondidos por cambios en las relaciones parentales o de lo contrario empezarán a producirse problemas que señalen esta dificultad.

La culminación del proceso de individuación es el punto clave en esta etapa y dependiendo de cómo ésta sea vivida y aceptada por el resto de la familia le será mas o menos facilitada al adolescente. En este apartado debemos centrarnos en la relación entre el subsistema parental y el hijo adolescente. Las características que deben de sostener dicha relación son las siguientes: un apego vivido adecuadamente, comunicación respetuosa, capacidad de resolución conjunta de problemas, y mutualidad como otra alternativa para llegar al entendimiento (Wynne, 11). Los recursos que necesita la familia habitualmente en esta etapa del ciclo vital para acrecentar su resistencia a las situaciones estresantes propias de la adolescencia son, según los estudios de David H. Olson (12) los siguientes: esfuerzos para conectar en cuestiones de personalidad, buena administración de las finanzas, y buena red de apoyo constituida por familia y amigos. Con respecto a los progenitores, dice este autor que es necesario que exista una buena valoración positiva de la relación conyugal y satisfacción con la vida. Como comentamos más adelante, los problemas que afectan a los padres en tanto tales (parentalidad), así como los que les aquejan como pareja (conyugalidad) van a tener su protagonismo en la presentación de dificultades del proceso natural de la individuación y la desvinculación.

La etapa de la adolescencia es una etapa de importantes cambios, desetabilizadores por cuanto se prepara la salida organizativa y emocional de uno de los miembros de la familia, y ésta debe estar dispuesta a ello; debe existir un equilibrio entre explorar lo novedoso y mantener la seguridad de lo conocido. La disponibili-dad hacia el cambio instala a la familia en una vía sana. Cualquier elemento, personal, parental, conyugal, que 
ORIGINALES Y REVISIONES

cuestione dicha disponibilidad, podrá actuar como un germen reforzador de los comportamientos desafiantes y provocadores del adolescente que, al cronificarse, hagan que se manifieste a través de los problemas que llamamos trastornos de comportamiento.

\section{4.- La elección del abordaje estructural}

La afirmación de S. Minuchin (13) de que la estructura del contexto donde nos desenvolvemos condiciona la expresión de nuestra conducta, es el punto de partida de lo que se ha dado en llamar escuela o enfoque estructural. Dice Minuchin (13) que las personas "en sus interacciones solo manifiestan parte de sus posibilidades. Estas son múltiples pero solo algunas son sacadas a la luz por la estructura del contexto". Desde esta perspectiva, un síntoma es producto de un sistema familiar disfuncional en el que si la organización familiar se vuelve más "normal" el síntoma desaparecerá. La disfunción es entendida como un desorden en la estructura familiar. No procede entrar aquí a describir la concepción minuchiniana de la estructura de la familia, aunque sí nos detendremos en analizar el funcionamiento de un subsistema que está involucrado, especialmente, en los trastornos de comportamiento de los adolescentes, el subsistema parental. Dice este autor que las funciones del holón parental (el formado por los padres y los hijos, en su interacción) son la crianza y la socialización de los hijos. Dentro de este subsistema los hijos aprenden la función de la autoridad (como elemento positivo de guía y protección), así como todo lo relativo a los cuidados, la protección, la socialización y el fomento de su crecimiento. La característica más permanente del subsistema parental es el continuo conflicto con el control, debido al continuo crecimiento del niño. Entre las observaciones de Minuchin sobre la estructura familiar que está detrás de la aparición de una conducta sintomática destacamos todo lo relacionado con la asunción de funciones por elementos de la familia que de forma natural no debían ejercerla (parentalizaciones), así como las coaliciones intergeneracionales, las perversiones jerárquicas, las fusiones simbióticas o el desapego extremo y los conflictos conyugales que se expresan en el subsistema parental.

La aparición de un trastorno de comportamiento en un joven será explicable en la medida en que la estructura del contexto donde vive no permite la aparición de otras conductas funcionales. Queremos imaginar que cuando el chico comienza a manifestar problemas (provocaciones, amenazas, agresiones, etc.) la familia pondrá en marcha sus propios recursos para intentar resolverlos. La elección de determinadas soluciones, y no de otras, tendrá relación con la dinámica de funcionamiento de la organización, de forma que, una organización funcional, que pueda leer los problemas como formando parte del proceso de desvinculación, será capaz de solventarlos y adaptarse. En cambio, una organización con problemas en su estructura, generará soluciones que probablemente tiendan a reforzar los problemas, convirtiéndose dichas soluciones, como dice Watzlawick, en los problemas. Por tanto, cualquier intervención terapéutica que se enfoque sobre la estructuración funcional del contexto familiar y que apunte hacia la continuación del proceso de desvinculación que se ve interrumpido por el trastorno de comportamiento, en 
teoría deberá hacer que los problemas desaparezcan. El cambio en la estructura familiar posibilitará que aparezcan comportamientos más funcionales y quede desbloqueado el proceso de desvinculación.

Lo que caracteriza la intervención estructural es la idea de que los límites entre los subsistemas no son nítidos, o que algunos individuos están participando en niveles de poder y de decisión que no les competen, siendo los padres u otras personas con ascendiente de autoridad sobre la familia los ql\}e están sustentando de alguna forma este tipo de estructura. El enfoque se dirigirá por tanto, a conocer qué particularidad tiene esa organización familiar, y a intentar cambiarla.

Resulta igualmente evidente que los jóvenes fuertemente afectados por el contexto familiar, a su vez afectan al contexto del que forman parte, creando presiones y tensiones que harán que los demás miembros de la familia se vean emocionalmente expuestos a estas situaciones. Cancrini llega un poco más lejos al afirmar con rotundidad que la expresión sintomática forma parte, como un todo, de la disfunción familiar (6). Así, este autor habla de la correspondencia que hay entre el tipo de problema emergente en el individuo y el tipo de disfunción familiar o el momento del ciclo vital de la familia en que esta se ha visto estancada. El problema manifiesto sería como la punta del iceberg, bajo el cual se encontraría, fuera de nuestros ojos, la conflictiva correspondiente de la familia.

Por todo lo anteriormente expuesto, los autores llegamos a la conclusión de que la intervención familiar de tipo estructural sería la más conveniente para los jóvenes con trastornos de comportamiento que han llegado a nuestra consulta.

\section{5.- Procedimiento terapéutico}

La intervención estructural que realizamos en este tipo de casos pivota sobre los siguientes postulados y acciones:

$1^{\circ}$._ La hipótesis de que el síntoma cumple una función homeostática en el funcionamiento familiar

$2^{\circ}$._ La necesidad de lograr la confianza de la familia en nuestro quehacer, trabajando mucho sobre el establecimiento de una sólida relación de cooperación. Esto incluye la realización de alguna lectura de la conducta del chico, diferente de la que hace la familia (que solamente aprecia maldad o cree que el problema es debido a un trastorno de la personalidad) con el objetivo de que éste se sienta incluido y no rechace el proceso terapéutico. A veces, dependiendo de la problemática familiar y de la colaboración del adolescente en el proceso, señalamos la necesidad de realizar una atención individualizada, cuyo .objetivo es mantener un espacio propio. Esta indicación, que hemos realizado de una forma empírica, casi intuitiva, refuerza el proceso natural de distanciamiento del adolescente respecto de su familia al ofrecer un encuentro caracterizado por la posibilidad de la intimidad y la expresión y análisis de sus deseos y sentimientos, todo ello dirigido a facilitar el proceso de construcción de identidad.

$3^{\circ}$._ El intento de descubrir alianzas o coaliciones ocultas, o de identificar las pau- 
tas o secuencias conductuales, habitualmente repetitivas, de los miembros de la familia que se ven involucrados alrededor del síntoma.

$4^{\circ}$.- El trabajo de reconstrucción de un modelo de autoridad parental compartida. La hipótesis que nos mueve a hacer esta labor está sustentada en nuestra experiencia de que cuando empiezan los problemas y tensiones en la relación padres-hijo, es justamente el desacuerdo entre los padres sobre el ejercicio de la autoridad uno de los factores que retroalimentan y refuerzan el mantenimiento del problema. Para conseguir este objetivo trabajamos, durante una parte de las sesiones, con los padres a solas. De esta forma, por un lado se delimita nítidamente el subsistema que forman los cónyuges en tanto padres que comparten autoridad y responsabilidades comunes sobre los hijos. Por otro lado, empezamos a trabajar con los demandantes y señalamos la necesidad de responsabilidad e implicación a un nivel de igualdad entre los padres (por tanto, estructural), en el subsistema parental. Trabajar con los demandantes supone hacerlo con quién más está sufriendo por el problema y por tanto con quién más deseo tiene de lograr cambios. Esto no quiere decir que no se vayan a plantear dificultades para cambiar sus actitudes o conductas, pero sí que van a poner una mayor disponibilidad de recursos para intentar los cambios. Por tanto, trabajamos con quienes tienen claves decisivas para lograr el cambio. En efecto, ya Bowen (14) comentaba que sus mejores resultados con niños o adolescentes con problemas los había logrado cuando, partiendo de la premisa de que todo el sistema familiar cambiaría si cambiaba la relación conyugal, comenzó a pedirles a los padres que dejaran al hijo en la casa y se concentraran en sus problemas personales. No tan atrevidos como este autor, por cuanto un señalamiento de este tipo podría suponer un mensaje inculpatorio hacia los padres y por tanto levantar una enorme resistencia, el contexto del encuentro que nosotros les proponemos es definido como el de compartir con los terapeutas aspectos que conciernen a sus preocupaciones sobre la conducta del hijo que no pueden expresarse ante este, así como de asesoramiento sobre su propia actuación en relación con el joven. Esta alianza explícita con los demandantes en general ha sido bien aceptada por los chicos siempre y cuando ellos hayan percibido en este acto nuestra intención de ayudar a sus padres ante estos problemas. En el espacio que tenemos con los padres subrayamos la necesidad de compartir un modelo de autoridad común frente al chico, de trabajar como en un equipo y, sobre todo, de no desautorizarse entre ambos ante él. Si la respuesta de los padres es coherente y firme, las provocaciones del joven tenderán a ir disminuyendo. A veces los chicos cuestionan su necesidad de acudir a las sesiones, cuando saben que hay un espacio donde se está decidiendo sobre como conducirse con ellos. Pero si los padres han conseguido establecer el modelo de autoridad compartida, generalmente logran que los chicos acudan a las entrevistas. En caso contrario, estaremos en condiciones de que declaren abiertamente su tremenda dificultad para establecer criterios comunes.

A. es una chica de 13 años con fracaso escolar, desobediencia a las normas domésticas, que se opone abiertamente a lo que los padres pretenden de ella, mentirosa, peleona, siempre está con chicos en vez de con chicas, y tiene la habilidad de sacar de las casillas a sus padres. La demanda se presenta a raiz de los malos resultados en la escuela y de su provocación constante hacia los profesores (los padres han recibido múltiples 
quejas). Es la mayor de dos hermanas. La hora de comer es temida en la familia. Según comentan los padres, durante la comida se dedica a fastidiar a la hermana con bromas difíciles, o no sigue las normas de conducta de la mesa. La madre empieza a reñirle y a intentar corregirla, hasta que se echa a llorar por impotencia. Cuando llega el padre y ve a la mujer llorando se le quitan las ganas de comer y se enfurece con A., hasta un punto en que parece que le va a pegar (según reconoce la madre, nunca le ha puesto una mano encima); entonces A. se tira al suelo y se pone en posición fetal, como defensiva frente a lo que el padre puede llegar a hacerle. La madre interviene enfrentándose al padre para evitar que ocurra lo temido: que el padre se descontrole y le pueda hacer daño a A. Ante esta escena que se repite casi diariamente, le decimos a los padres que esto es como una sinfonía, donde A. pone la música y ellos interpretan la danza. Bailan al son que les marca. Hasta que nosotros hacemos esta lectura los padres no habian tomado conciencia de cómo dejaban en manos de A. la dirección de este "drama" musical. Nuestra propuesta es clara: ellos deben tocar la sinfonía y la chica danzarla al son que ellos marquen. Para lo cual será preciso que seleccionen una nueva estrategia de comportamiento frente a la conducta de $A$.

Cualquier tipo de intervención dirigida a conseguir establecer un modelo de autoridad compartida entre los padres será de utilidad para lograr cambios en el problema. En este sentido, solemos aprovechar las anécdotas que nos cuentan las familias para construir sobre ellas los cambios de comportamiento que deberán experimentar los padres con el fin terapéutico. En el caso de que los padres manifiesten abiertamente una clara dificultad para ponerse de acuerdo, al menos ante el chico, optamos por trabajar sobre lo que nos permita comprender su origen. En general, este trabajo ayuda a que cada cónyuge comprenda mejor la dificultad actitudinal del otro, y puede servir para ayudarlo a modificarla.

F. es un chico de 12 años, segundo de tres hermanos, de una familia donde el padre se nos presenta deprimido ante su dificultad de controlar la conducta del menor. La madre dice que está histérica porque tampoco sabe como controlarlo. Niño desobediente, respondón, no hace caso de ningún mandato materno y últimamente tampoco del padre. El día que tenían que venir por primera vez a la consulta se escapó de casa y tuvieron que estar buscándolo por todo el pueblo hasta que dieron con él. El padre dice que es incapaz de pegarle una torta a su hijo porque a él su padre le pegó mucho y no cree que sea el método más conveniente de educar al hijo. Su esposa piensa que darle un cachete no es malo, pero no quiere contrariar el criterio educativo de su marido.

En el caso comentado, la dificultad por establecer el tipo de castigo ante el chico viene determinada por la experiencia paterna del castigo físico. Si analizamos con detalle lo que esto significa, podremos apreciar cómo la negativa del padre a aceptar la posibilidad de dar un cachete al hijo supone que el padre está recordando y reactualizando en su relación con él lo ocurrido con su propio padre cuando él era un niño. Sin duda un proceso donde el recuerdo modula de tal forma la respuesta actual que puede llegar a anular la capacidad de percepción del padre sobre el momento presente con su hijo propio. Es un proceso de proyección de un recuerdo traumático de la propia historia en la historia presente del chico. En efecto, lo que el padre no quiere es que su hijo sienta el dolor y lo que 
él mismo sintió con respecto al suyo. Conviene en este caso aclarar al padre que el momento presente tiene sus propias particularidades. Son otras personas y otras circunstancias, que requieren, por tanto, diferentes respuestas adecuadas a ellas.

Algunos padres manifiestan abiertamente que ellos no son culpables de lo que le sucede al hijo. Es evidente que el tipo de intervención, que enfatiza la necesidad de cambio en la diada parental, implícitamente invita a sentir esa culpabilidad. Generalmente respondemos que no se trata de culpabilizarlos por cosas que han ocurrido en el pasado y que son indemostrables. Sin embargo, para el hijo "síntoma" es preciso que ellos cambien algunos comportamientos o actitudes que presumiblemente le ayudará a crecer. Se trata, por tanto de resposabilizarlos no frente al pasado, sino ante el futuro.

También es el momento en que se ponen de manifiesto las dificultades de los padres por mantener el modelo de autoridad compartida que hemos establecido. En realidad, estas dificultades hablan más de la relación de pareja que de no haber entendido o comprendido nuestro mensaje. Y esta información, que a nosotros nos cuesta varias entrevistas conocer, el chico la conoce desde siempre. De ahí que, si la función del síntoma es evitar una confrontación conyugal, el chico continúe atado (triangulado) a través de su renuncia al crecimiento, a los padres. Como veremos en el siguiente apartado, en algunas ocasiones hemos llegado a tiempo de proponer un trabajo en profundidad sobre las dificultades individuales de los padres para acercarse al modelo de autoridad compartida, y a estructurarse de forma funcional, pero en otros casos la familia ha abandonado el tratamiento antes de que lo logremos.

Perdimos el caso de K. un chico de 14 años, hijo único de una pareja joven, con un trastorno de comportamiento consistente en desobediencia, insultos y amenazas de agresión a los padres, sobre todo a la madre, y fracaso escolar por el mal comportamiento (se le había expulsado muchas veces de clase por distraer la atención de los demás alumnos). Iniciamos el trabajo estructural con la familia y mejoró parcialmente la situación. Notamos que el padre no estaba de acuerdo con muchas de las exigencias de comportamiento de la madre hacia K., so pretexto de que eran demasiado duras para el chico. lgualmente observamos cómo el padre era más afectuoso con el hijo, que la madre, y compartía con el ratos de ocio y juegos. Eran dos contra una. En la tercera sesión nos cuentan que la madre había decidido no complacer al hijo con los regalos de Reyes, por no haberse comportado correctamente, como ella esperaba, y el padre, le dio a $K$, lo que él quería: unas botas. Quedó demasiado en evidencia el desacuerdo conyugo-parental, y no estuvimos atentos a poder reformular que el problema estaba situado en esa órbita y no sólo en la del comportamiento del chico. En este caso nos olvidamos que la demanda fue presentada por la madre, que necesitaba que alguien refrendara lo que ella creía que era correcto hacer con el chico, sin percatarnos que aceptar su petición era colocarnos, implicitamente, contra la opinión del padre, al menos en lo relativo a la manera de establecer los límites que el chico parecía estar pidiendo a voces.

Con este tipo de trabajo, hacia la tercera o cuarta entrevista puede haber remitido el comportamiento que motivó la demanda; puede ocurrir que aparezcan otras demandas o que se reformulen situaciones que antes no aparecían claras para nosotros. Y también 
pueden desaparecer los casos. Si la remisión sintomática es prácticamente completa entonces hacemos alguna entrevista de seguimiento entre cuatro y ocho semanas después para reevaluar el mantenimiento de los cambios y luego otra a más largo plazo antes de dar el alta definitiva.

\section{6.- Obtención de la información para el análisis}

El análisis que hemos realizado sobre los casos que presentamos en este trabajo se ha efectuado sobre la base de la información que hemos recopilado a través de dos medios: Por un lado, en nuestra metodología de trabajo incluimos habitualmente la grabación en vídeo de todas las sesiones. Por otro lado, uno de nosotros siempre observa la sesión a través de un circuito cerrado de televisión y toma notas sobre lo que sucede, con lo cual al final de cada entrevista hacemos un registro manuscrito con todo ese material. La revisión de las grabaciones y de las notas tomadas ha sido la principal fuente de información de este trabajo.

Finalmente, hemos realizado un seguimiento telefónico para conocer en que situación se encontraban las familias y sus hijos. Este seguimiento se ha realizado, dependiendo de la fecha de conclusión del tratamiento de cada familia, entre los 6 y 12 meses o más tras el alta o el abandono. Según las repuestas que nos dieron las familias (hablábamos con alguno de los progenitores) pudimos hacer tres grupos: los que habían evolucionado favorablemente, los que no habían cambiado y los que habían mejorado un poco pero todavía presentaban alguna sintomatología (mejorías parciales).

\section{7.- Análisis de los casos estudiados en función de los resultados.}

\section{1.- Descripción de los casos.}

Hemos intervenido con las técnicas estructurales en un total de 16 casos de niños y adolescentes de edades comprendidas entre los 11 y los 20 años (media 14,6, mediana 15), once chicos y cinco chicas (ver Tablas 1 y 2). Agrupando los comportamientos sintomáticos o diagnósticos, según el grado de angustia que producía en los demandantes, se distribuían de la siguiente forma: había dos casos con diagnóstico de psicosis infantil, un caso de bulimia, uno de anorexia, uno de hiperactividad, uno con un trastorno de angustia acompañado de una conducta regresiva y los otros diez casos de problemas de rebeldía, que en mayor o menor medida se acompañaban de trastornos agresivos heterodirigidos (excepto en un caso que amenazaba con suicidarse), fracaso escolar, fugas o aislamiento. En dos casos la familia era monoparental, y en otro reconstituida, el resto estaba formado por los padres del paciente y su prole, siendo el promedio de número de hijos de dos (rango entre 1 y 3 , mediana y media 2). De los dieciséis casos tratados, 10 han tenido una evolución favorable, con remisión sintomática completa (lo que supone un 62,5\% de los casos)(ver Tabla $\mathrm{n}^{\circ} 3$ ). Dos han tenido una evolución claramente desfavorable (12,5\%), en uno de los 
casos con abandono del tratamiento. Y en los otros cuatro casos (25\%) ha habido mejorías parciales aunque sin remisión clínica. Entendemos como mejoría parcial una apreciable reducción de los síntomas o comportamientos problemáticos o una mejor relación del paciente con sus padres que hace que la convivencia resulte menos conflictiva. No obstante, en estos pacientes persistirían comportamientos sintomáticos más llevaderos desde el punto de vista de la familia.

Tabla $n^{\circ} 1$

VARIABLES CUANTITATIVAS

\begin{tabular}{|l|c|c|c|}
\hline EDAD & MEDIA & MEDIANA & RANGO \\
$11-20$
\end{tabular}

Tabla $\mathrm{n}^{\circ} 2$

VARIABLES CUALITATIVAS

\begin{tabular}{|l|c|}
\hline SEXO: & 11 \\
- VARONES & 5 \\
- MUJERES & \\
\hline TIPO DE FAMILIA: & 13 \\
- AMBOS PROGENITORES & 2 \\
- MONOPARENTAL & 1 \\
\hline RECONSTITUIDA & \\
\hline DIAGNÓSTICOS: & 2 \\
- PSICOSIS INFANTIL & 1 \\
- BULIMIA & 1 \\
- ANOREXIA & 1 \\
- HIPERACTIVIDAD & 1 \\
- ANGUSTIA-REGRESIÓN & 10 \\
FUGASESIENCIA, DESAFIO, AGRESIVIDAD, & \\
\hline
\end{tabular}

Tabla $n^{\circ} 3$

TIPO DE EVOLUCIÓN

\begin{tabular}{|l|c|c|}
\hline & $\mathrm{N}$ & $\%$ \\
\hline FAVORABLE & 10 & 62,5 \\
\hline DESFAVORABLE & 2 & 12,5 \\
\hline MEJORIAS PARCIALES & 4 & 25,0 \\
\hline
\end{tabular}




\subsubsection{Análisis de los casos; perspectiva individual}

Como ya señalamos al principio de este trabajo, hemos centrado nuestras observaciones a nivel individual sobre los elementos o factores que ayudan a definir la propia identidad, buscando diferencias significativas entre aquellas características que presentan los pacientes en los casos que evolucionan favorablemente frente a los demás. Evidentemente el pequeño tamaño de la muestra en estudio no aconseja la realización de pruebas estadísticas, pero no obstante vamos a señalar las diferencias que hemos hallado y que, a la luz de nuestra base teórica permitirían hacer inferencias sobre los resultados.

La evolución favorable en el tratamiento está claramente asociada a que el paciente sea capaz de sostener ante sus padres sus propios deseos en lo relativo a su comportamiento $y$ al proyecto de su desempeño profesional, laboral y social como adultos. En efecto, en todos los casos con buena evolución terapéutica hemos encontrado que los pacientes no tenían una especial preocupación por cumplir con las expectativas que alguno de sus progenitores había puesto sobre él. En la mayoría de los casos (9 de 10) el chico es protagonista de sus propios deseos porque los padres no interfieren en ellos o, dicho de otro modo, las expectativas de los padres sobre lo que deben desear y hacer sus hijos no son de tal intensidad que, si son rechazadas por el hijo, cuestionen el afecto o el reconocimiento que los padres les tienen.

El cuestionamiento del respeto a las figuras de autoridad, característica frecuente en esta época de la vida, no fue manifestada abiertamente en los pacientes que tuvieron evolución favorable (lo cual no quiere decir que no experimentaran esa clase de sentimientos o fantasías). Entendemos que ello habla de una cierta capacidad de autoprotección. Solamente en dos de los 10 casos de evolución favorable encontramos una dificultad para mantener los límites de la intimidad, pero no en un sentido agresivo, sino más bien de llamada de atención (consumían alcohol o drogas, o tenían amigos consumidores o problemáticos y no tenían reparos en comunicarlo a sus padres, que alarmados, tendían a controlarlos aún más; control del que luego se quejaban los pacientes).

En los dos casos con evolución desfavorable, los pacientes habían empeñado su existencia precisamente en contrariar esas expectativas que tenían los padres, aplicándose a fondo en frustrarlos. Es necesario aquí enfatizar que cuando hablamos de expectativas de los padres, lo hacemos desde la perspectiva de que los padres sólo reconocerán al hijo si actúa conforme esperan, pero no será reconocido (confirmado) si se sale del patrón previsto. Es obvio que los padres depositan expectativas sobre la realización personal de sus hijos. Lo que hace diferente a esa expectativa y la convierte en patológica, es que no se puede defraudar, ya que alguno de los progenitores, $o$ ambos, ha decidido que lo que él no logró o no tuvo (incluyendo las atenciones o los afectos de los que careció en su infancia), lo ha de realizar o tener su hijo. Con lo cual se le hurta al chico la posibilidad de decidir que es lo que él quiere, que es lo que él desea. Además, esto habitualmente coloca al chico en una posición paradójica: si él hace lo que esperan los padres contraviniendo quizás sus propios deseos, entonces se sentirá mal. Pero si él hace lo que desea, entonces los padres no lo confirmarán, y entonces también se sentirá mal. Esa "expectativa" tramposa se da sobre todo en los casos en que los padres depositan en la persona del chico y en su vida, proyectos personales que les atañen a 
ellos. De los cuatro casos en los que hubo mejorías parciales, pero no remisiones clínicas, en tres de ellos, pudimos encontrar esta situación, mientras que sólo lo pudimos observar en uno de los diez que habían evolucionado favorablemente.

M. es una chica de 16 años, obesa, la mayor de dos hermanos cuyos padres están muy angustiados porque han notado que la niña come a escondidas y que no tiene ningún tipo de control frente a la alimentación. La madre sobre todo está preocupada porque $M$., cuando ve que no puede ponerse un vestido hace una especie de dieta que consiste en no comer casi nada y pierde en poco tiempo 15 o 20 kilos, que inmediatamente después vuelve a coger porque cuando se ve bien no puede refrenar su impulso de atiborrarse. La preocupación del padre es que ha notado que come a escondidas y que miente cuando la descubren. La madre es una mujer que lleva toda su vida haciendo dieta. Ella de jovencita también era obesa y a base de mucho sacrificio consiguió adelgazar y aun hoy se mantiene delgada a base de dietas estrictas. Le da mucha pena ver que su hija se compra ropa que al poco tiempo no se puede poner.

En este caso es posible apreciar cómo el síntoma de la hija es un "recuerdo" de los problemas alimentarios de la madre. La expectativa de la madre era que su hija no sufriera lo que sufrió ella cuando era adolescente obesa. Y le propone a su hija un modelo de alimentación que debe ser deseado por ella misma. Es decir, no le deja espacio a M. para que ella decida si se acepta obesa o no, si es su propio deseo. M. decide complacer a la madre, en la medida que hace una dieta, pero entonces ila hace porque ella quiere o porque el deseo es de su madre?. La única forma de saberlo es no seguir la dieta, comer hasta atiborrarse, pero cuando está gorda también se siente mal, porque no se gusta así. Por tanto se encuentra atrapada sin saber si lo que ella hace obedece a sus propios deseos o a los de su madre. En esta situación extrema, la paciente lo que trata es de descubrir cual es su propio deseo, porque todavía no ha sido posible que se reafirme en él. Su identidad, pues, está cuestionada. El caso narrado es uno de los cuatro que dejaron el tratamiento con una mejoría parcial, pero sin una remisión completa.

Emplearse en contrariar de forma visceral las expectativas paternas también impide que el adolescente pueda definirse a sí mismo a través de sentirse protagonista de sus deseos, dado que sus comportamientos no son decididos con libertad. Los chicos actúan sin pensar, siquiera, en su propia conveniencia.

$\mathrm{Si}$ analizamos la asociación entre la evolución y la presencia de indicadores personales de que el paciente guarda para sí algunas fantasías, pensamientos, sentimientos o actos que no comunica a sus familiares, encontramos que en los dos casos con evolución desfavorable, no existen estos límites para su intimidad. Así, los pacientes hablaban abiertamente de sus sentimientos hostiles hacia los padres, fantaseando con actos de venganza por los castigos recibidos, insultándolos abiertamente, desafiándolos a hacer aquello que amenazaban. Resultaba llamativo, y añadiríamos que dramático, en comparación con los demás casos, cómo los pacientes trataban a sus padres como iguales en una disputa, añadiéndole además amenazas, de estilo similar a la que los padres podían proferir. En el caso ya comentado de K. este le decía a la madre que le iba a pegar; y al padre, que reuniría a los amigos de su pandilla y que lo cogerían de improviso para darle una paliza. Todo ello 
aderezado de insultos y fantasías de que en un futuro, cuando él tuviera más cuerpo, no iban a poder hacerle nada.

En tres de los cuatro casos con remisión parcial, también encontramos dificultades para el establecimiento de un espacio de intimidad, pero en estos, entre los que se encuentran los dos pacientes diagnosticados de psicosis infantil, el problema residía en la imposibilidad de que los chicos se manejaran con la contingencia de poseer un espacio personal propio. En efecto, estos chicos (una niña de 11 años y un chico de 13) no tenían ningún tipo de filtro para comunicar pensamientos, sentimientos e ideas a sus padres, progenitores que además se caracterizaban por manifestar que sabían exactamente que es lo que pensaban sus hijos, antes incluso que ellos lo pudieran decir. En estos casos, el proceso de individuación, entendido como la posibilidad de haber establecido relaciones con cierto grado de intimidad con personas ajenas a la familia (por ejemplo con pares) no se había producido. Entendemos que ello implica un menor grado de desarrollo de sus capacidades y de sus habilidades para afrontar las dificultades de la adolescencia e, igualmente, una menor capacidad de los padres para conducirse ante estos cambios.

Por último, la transgresión de las normas familiares fue una característica común a casi todos los casos, y precisamente uno de los más frecuentes motivos de consulta. Por tanto, la transgresión, desde la perspectiva individual, no supone un indicador sobre la evolución de los pacientes aunque, como veremos en el análisis de la estructura familiar, el significado que le da la familia si resulta diferente, y consecuentemente la forma de tratarla.

\subsection{2.- Análisis de los casos: perspectiva familiar}

No hay datos que permitan asociar la evolución de los casos con la composición de la familia. La característica común a todos ellos era que la demanda de tratamiento estaba sustentada por los padres sobre la base del grave problema de convivencia en el hogar que suponía la presencia del chico con sus trastornos de comportamiento. Sin embargo, en el momento en que inquiríamos sobre el modelo de autoridad con el cual los padres estaban abordando la crianza de este hijo, excepto en un caso, aparecía nítidamente un desacuerdo entre ellos acerca de la forma de actuar sobre el mismo. Básicamente este desacuerdo consistía en la diferencia de significados que le daban al comportamiento sintomático, y por tanto, el diferente trato que sostenían que había que dispensarle al chico. En general, uno de los progenitores se muestra comprensivo con el comportamiento, que entiende como resultado de un conflicto interno o de problemas del chico, mientras que el otro piensa que es un problema de falta de disciplina que requiere algún tipo de correctivo. Y estos padres se encuentran atrapados de forma que, o bien cada uno de los dos actúa según sus propios criterios y por tanto se ven abocados a tener que discutir periódicamente con el otro cónyuge a causa de ello. O bien el que piensa que con disciplina se arreglaría, opta por no actuar. En cuyo caso, y desde la desesperación por que todo sigue igual, periódicamente es demandado por el otro progenitor para actuar. Esta actuación solicitada, generalmente es después boicoteada, por temor a que suponga un mal mayor. Con lo cual, en todos los casos, menos en uno, hemos comprobado como los padres, de forma más o menos abierta, admiten discrepancias y/o discusiones frecuentes, por este motivo. Dado 
que esta conflictividad se presentó en casi todos los casos no supone ningún indicador que discrimine la evolución postratamiento.

En la medida en que uno de los progenitores se sitúa en una posición comprensiva con el hijo-problema se produce un fenómeno de refuerzo o tolerancia del comportamiento sintomático. Algunas madres o algunos padres relacionan el trastorno de comportamiento de sus hijos bien con problemas del desarrollo de su personalidad, bien lo pueden ver como el resultado de afrontar inadecuadamente conflictos extrafamiliares (amistades, novias, la escuela, uso de sustancias, etc.) o también como fruto de una incorrecta forma de trato por parte del otro progenitor. Ello no obsta para que sientan que el comportamiento del chico es doloroso y disfuncional para la marcha de la familia. Si dicha posición de comprensión tiene utilidad para regular la distancia interaccional o la calidad de la relación con otros miembros de la familia, por ejemplo con el otro cónyuge, es algo que no hemos podido constatar en todos los casos. Ni en principio, en los casos en los que lo hemos detectado, ello parece tener relación con la evolución. Sí que resulta claro que la aparición del síntoma supone el principal problema de la familia y por tanto un acercamiento relacional de los padres (los acerca a pesar de que mantengan desacuerdos o posiciones encontradas).

Otro elemento común observado en todos los casos es el permiso o la tolerancia de uno de los progenitores, y con menor frecuencia de los dos, hacia la transgresión de las normas de convivencia familiares. Como se comentó al principio, la transgresión es el camino para la adquisición de nuevos espacios de autonomía, y por tanto es útil para la construcción de la identidad. Si el chico sobrepasa un límite, apoyado por el poder de su progenitor aliado y ante la impotencia del otro padre, la transgresión no tiene utilidad para construir identidad. Si el chico no siente que lo que obtiene es "conquistado" al poder de sus padres, entonces no puede saber si lo que hace es por su propio deseo o por el de los padres. De ahí que necesite ir aumentando el grado de la transgresión hasta que los padres presenten un "frente" y aparezca un límite sólido ante el cual definirse. La inexistencia o la "porosidad" de un límite no le permite al chico poder definirse frente a él. Si los padres van cediendo terreno sin oponer resistencia, entonces el chico no sabe si lo logrado le pertenece a él (le sirve para construir su identidad) o les pertenece a ellos (la identidad de los padres).

$M$. es un adolescente de 16 años, que desde hacía unos meses amenazaba a los padres con marcharse de casa e irse a vivir con unos "ocupas". En algunas ocasiones se había escapado durante periodos cortos de tiempo. Fracaso escolar, relaciones con una pandilla de chicos de aspecto marginal y estética provocativa, eran otros elementos de su cuadro "sintomatológico". Es el único hijo de una familia reconstituida formada por una madre divorciada (el padre de M. tiene una Psicosis Esquizofrénica) y un padrastro, también divorciado, que tiene un hijo un poco menor que M., pero que vive con su madre. Ambos cónyuges son docentes y conviven juntos desde que el niño tenía 3 años. A la primera consulta se presentan los tres, el chico vestido informalmente y con un corte de pelo un poco especial: se había afeitado toda la cabeza excepto la parte de la nuca. Ninguno de los padres estaba de acuerdo con este atuendo ni pelado, y estaban desmoralizados sobre como actuar con él. M. también consumía porros de vez en cuando y los fines de semana volvía a 
ORIGINALES Y REVISIONES

casa a altas horas de la madrugada. La preocupación de los padres estaba centrada en la actitud desafiante y provocativa que el chico mantenía con ellos dentro del hogar.

En este caso pudimos apreciar cómo el chico había ido paulatinamente haciendo cosas que cada vez lograban incomodar más a los padres. Estos, so pretexto de una actitud beneficiosa para la crianza, la tolerancia, habían ido cediendo terreno respecto a sus criterios estéticos y de comportamiento, lo cual había obligado a $\mathrm{M}$. a buscar cual podía ser el límite de esa tolerancia, incluso a pesar de que el corte de pelo que se había dado le parecía horroroso (él mismo nos lo confirmó posteriormente). Sólo cuando encontró una barrera familiar sólida, empezó a comportarse y vestirse de una forma más adaptada a su contexto. Los "síntomas", por tanto, sólo eran una forma de sentirse, y por tanto de ayudarse a definir su identidad. Cuando los padres comenzaron a hacer cumplir sus criterios, entonces M. pudo optar por realizar transgresiones menos "aparatosas" que le permitieron sentirse él mismo.

El hecho de que la desobediencia a los límites fuera tolerada por uno de los padres nos permitió aventurarnos a conocer cual era la base biográfica que hacía que este padre no pudiera hacer respetar las normas familiares con ese hijo en concreto. Así encontramos una amplia gama de problemas "arrastrados" desde sus adolescencias que en este momento se estaban reactualizando en la relación con sus hijos. Haber tenido padres excesivamente severos, autoritarios y rígidos, conllevaba una enorme dificultad para que ellos se identificaran con la parte positiva que tiene la autoridad para el crecimiento. En tres casos nos encontramos con padres o madres que quedaron huérfanos siendo niños o jóvenes y que se criaron en ambientes con carencias de tipo afectivo. En otros cuatro casos la desvinculación de los padres de sus familias de origen no había concluido exitosamente, o había sido muy traumática (expulsados del hogar, enfrentamientos todavía no cerrados). Por último, había otro grupo de progenitores caracterizados por sentir que no estaban realizados como ellos habían proyectado (en el ámbito profesional y laboral). Todo lo cual supone que en el proceso de desvinculación de los hijos se están moviendo sentimientos en los padres que al ser proyectados pueden producir un fenómeno paradójico. En efecto, al tratar a sus hijos según el criterio de "así es como me deberían haber tratado a mr", están ignorando las necesidades reales de los chicos, con lo que no los están percibiendo tal cual son: se produce entonces una especie de ignorancia de ciertos aspectos de su yo, dificultándose por tanto su proceso de construcción de la identidad. Igualmente, esa proyección de la historia de un progenitor sobre un hijo permite que éste tolere la transgresión a las normas porque en realidad se la está permitiendo a sí mismo. Ambos fenómenos familiares, proyección - ignorancia del yo del chico - y tolerancia a la transgresión, van a conllevar que éste necesite amplificar considerablemente los comportamientos "patológicos" con una intención claramente sana: saber quien es él.

No hemos encontrado relación entre la evolución postratamiento del paciente y estos fenómenos que acabamos de comentar. Probablemente, bajo una emergencia similar (ya hemos comentado que en casi todos los casos los padres reactualizaban sus conflictos adolescentes en el momento de la adolescencia de sus hijos) pudiera ser diferente el grado de desvinculación alcanzado por estos padres con respecto a sus propias familias de ori- 
gen, lo que podría explicar el diferente peso específico sobre la aparición del problema en el chico (tal como sugiere Cancrini), así como sobre el pronóstico. El alcance de este trabajo no nos ha permitido profundizar en estos aspectos, que no obstante quedarían pendientes de una investigación más específica.

Respecto al tipo de interacción que mantienen los miembros de la familia ante nosotros, sí hemos encontrado que puede constituirse en un indicador de evolución. En efecto, en los dos casos con evolución desfavorable, los pacientes sostenían en nuestra presencia una posición de desafío, falta de respeto e insultos o amenazas hacia uno de los padres o los dos. También coincidieron en que expresaban más fácilmente su emotividad. La respuesta familiar a este fenómeno era de una extrema pasividad, circunstancia que entendimos dentro del contexto de la consulta como una demostración de lo mal que estaba el chico, y de lo impotente que se sentían los padres. En los casos en que la evolución ha sido favorable, los chicos no manifestaban verbalmente los probables sentimientos de rabia que les producía lo que estaban narrando los padres sobre su comportamiento, aunque podían notarse gestos o caras de fastidio. Incluso en los casos en que el enfado era demasiado evidente, en ningún momento se producían faltas de respeto hacia los padres, aunque sí reproches.

Por último, el único factor de la órbita familiar con una clara influencia negativa sobre la evolución terapéutica ha sido la presencia de familiares de la familia extensa, con un peso específico enorme sobre la conducta del chico. En los dos casos con evolución desfavorable encontramos una alianza del chico-problema con un tío o un abuelo que se podía permitir descalificar la autoridad de los padres, sin que por parte de estos hubiera una respuesta nítida de defensa frente a esta intromịsión. En efecto, si los padres intentaban poner en marcha un modelo de autoridad responsable y compartida, el chico podía seguir transgrediendo porque encontraba refugio en alguno de esos familiares que no sólo gozaban de autoridad y prestigio para el chico, sino también para sus padres. Estos, en vez de enfadarse o enfrentarse a estas descalificaciones, parecían aceptarlas con resignación, e incluso con sentimientos de culpa. Ello nos parece un indicador de que estos padres todavía continúan manteniendo ataduras emocionales, y de alguna forma incluso organizativas respecto a sus familias de origen, y por tanto que no han resuelto aún su propio proceso de desvinculación.

\section{8.- Discusión y conclusiones}

En los últimos años se ha producido una gran cantidad de literatura sobre el abordaje psicoterapéutico de los trastornos mentales y de comportamiento de los niños y adolescentes. Problemas como la drogodependencia, la violencia juvenil, la anorexia y la bulimia han recibido una enorme atención por los investigadores y los clínicos. En muchos de los trabajos consultados se pone énfasis en la necesidad de realizar abordajes familiares para este tipo de problemáticas, incluso con afirmaciones sobre las ventajas de este método terapéutico sobre otros $(15,16,17,18,19,20,21,22,23,24,25,26,27)$. Sin 
ORIGINALES Y REVISIONES

embargo, no existen muchos estudios en la línea de establecer si un tipo de abordaje familiar es más eficaz que otro para determinado tipo de síntomas o problemas.

El hecho de que haya situaciones clínicas que no se han podido beneficiar del tipo de intervención estructural que hemos construido, nos conduce a pensar en la posibilidad de que: 1) o bien no hemos implementado correctamente la intervención estructural nivel metodológico - ; o, 2) hay situaciones para las que la intervención estructural no es el mejor recurso terapéutico - nivel diagnóstico o de indicación —. La primera posibilidad nos resulta especialmente difícil de valorar dado que hemos protocolizado una intervención sobre todos los casos, que ha resultado útil en el 62,5\% de ellos. Obviando esta dificultad, nos parece especialmente interesante la segunda posibilidad, porque hablaría de la necesidad de realizar indicaciones terapéuticas ajustadas al tipo de problemática presentada y a los recursos de partida con los que cuenta el individuo y su familia.

Por ejemplo, Diamond y Liddle (28) propugnan un enfoque terapéutico familiar centrado en la reparación del vínculo de apego, dañado en los adolescentes con trastornos de conducta. Estos autores sostienen que la ruptura de la relación de apego sería la fuente de hostilidad y resistencia al cambio de conducta de los adolescentes. Sin embargo, los autores que propugnan una metodología terapéutica estructural sostienen que son precisamente las familias con patrones estructurales disfuncionales las que van a reforzar o mantener la conducta problemática del adolescente $(1,13)$.

En condiciones normales, la infancia tardía y la adolescencia son momentos especialmente críticos del ciclo vital individual y familiar. Desde la perspectiva individual, la necesidad de hacerse adulto implica romper con todo aquello que retrotrae a la persona a la infancia, adquirir responsabilidades más amplias, aceptar la disminución del grado de seguridad que ofrece la protección familiar, diferenciarse de la "masa del yo familiar" (Bowen), buscar identidad, y enfrentarse afectiva y emocionalmente al exterior de la familia, disminuyendo su grado de vinculación organizativa y emocional de ésta. Desde la perspectiva familiar, la adolescencia supone una crisis generada por la necesidad de un cambio en los roles parentales que deben redimensionarse de las funciones que cumplieron hasta ese momento: pasar desde el máximo control, protección y cuidado de la infancia a un grado de apertura suficiente como para ir permitiendo la vinculación del joven con el exterior. Los padres deben estar en condiciones de asumir un cierto riesgo, sin que la ansiedad les impida bloquear este proceso de salida de la familia. Además, esta situación hace que la familia deba adquirir una nueva organización en función de los cambios de roles que se están produciendo. En resumen, es posible hablar del Síndrome de la Adolescencia Normal, tanto desde el punto de vista individual (KNOBEL), como del familiar.

Qué factores, elementos o circunstancias convierten un problema de un adolescente, en principio "normal", en un trastorno de conducta es una pregunta de difícil respuesta. La literatura sistémica atribuye el síntoma a la disfunción familiar y los teóricos del apego, al daño de este vínculo. En nuestra casuística, hemos observado que todos los trastornos de comportamiento de nuestros adolescentes no pueden ser explicados por el mismo modelo etiológico. Si bien resultan evidenciables las características de la organización familiar descritas desde la perspectiva estructural, encontramos que en la aplicación del método terapéu- 
tico hay casos que no se resuelven. Y casos que sólo mejoran parcialmente. De lo cual inferimos que dicho modelo explicativo resulta, por sí solo, insuficiente. Por otro lado, los resultados positivos que hemos obtenido aplicando esta metodología nos conducen a continuar tomándola en consideración. De ahí nuestro interés en poder encontrar indicadores relacionados con la problemática presentada o con la estructura y funcionamiento familiar, que nos orienten sobre el recurso terapéutico más idóneo y eficaz. Sólo recientemente algunos investigadores de la terapia familiar se han planteado analizar la relación entre problemas específicos, técnicas de intervención, procesos terapéuticos y resultados (29).

Una gran parte de la bibliografía a nuestro alcance hace hincapié en la eficacia de la terapia familiar en los trastornos de comportamiento de los niños y adolescentes. Algunos autores (24) han demostrado en el terreno empírico que los problemas de conducta de los adolescentes están asociados a la presencia de coaliciones transgeneracionales, de forma que, tratando dichas coaliciones con terapia familiar, disminuye o desaparece la sintomatología. Así mismo, recientes trabajos han constatado que obtener cambios en el funcionamiento parental van aparejados de la reducción o desaparición de los problemas de conducta de los adolescentes $(24,30)$.

No obstante, no podemos perder de vista que los trabajos más recientes sobre el tratamiento familiar de los trastornos de comportamiento de adolescentes enfatizan la importancia del vínculo de apego dañado en su etiología y en cómo desarrollar los procesos terapéuticos para repararlo (tanto en el ámbito de las técnicas en sí, como en el de las actitudes de los terapeutas para conseguir sus metas)(28). Quizás un análisis más detenido de los casos desde este enfoque teórico puede aportarnos algunas explicaciones para entender los resultados desfavorables que obtuvimos con dos de ellos. Algunos autores (31) han encontrado que el fracaso terapéutico en este tipo de problemas parece estar asociado a que consciente o inconscientemente, el terapeuta había desafiado la imagen que la familia tenía sobre sí misma o sobre su comprensión del problema. Además éste había fracasado en el establecimiento de una relación empática con los padres. Pero también, varios estudios sugieren que la probabilidad de dejar el tratamiento se incrementa cuando durante las sesiones persisten intercambios negativos entre los padres y el adolescente, debido a que esto mantiene el recuerdo de experiencias negativas dentro de la familia (reduciéndose así las expectativas de cambio)(24,32,33). Diamond y Liddle (28) añaden que enfocar prematuramente el trabajo terapéutico sobre el control de la conducta del adolescente ignorando la valoración y reparación del vínculo de apego puede estancar el proceso terapéutico. Por tanto, en alguno de nuestros casos, el enfoque terapéutico que hace hincapié sobre el modelo de autoridad compartida pudo ser demasiado precipitado o bien no apropiado a sus características clínicas (tipo de problema emergente).

Entre los fenómenos de la órbita familiar observados en nuestra casuística y que parecen asociados con el proceso patológico de la adolescencia, los más destacables son: a) desacuerdos entre los padres sobre el modelo de autoridad a ejercer, lo que supone generalmente un permiso más o menos encubierto a la transgresión de normas familiares (implícitamente había un progenitor aliado con el adolescente). Y b) la presencia de personas de la familia extensa, con prestigio y autoridad ante los chicos y sus padres, que des- 
califican las actuaciones de éstos, sin que generen en ellos ninguna respuesta defensiva (coalición transgeneracional contra uno o ambos progenitores).

Desde la perspectiva individual, los elementos que parecen asociarse a que los problemas de comportamiento propios de esta edad viren hacia la patología son: a) el chico se encuentra, de alguna forma, involucrado en el subsistema parental, b) déficits en el proceso de individuación (problema presente en los dos casos de psicosis infantil y en la chica bulímica) y c) el empeño del chico en desafiar abiertamente y sin tregua la autoridad parental (posición simétrica en la relación, probablemente sostenida por la influencia de las personas de la familia extensa).

Wynne (11) señala que el apego sirve como fundamento del cual depende el éxito de otras conductas interpersonales esenciales (comunicación, resolución de problemas y mutualidad) de forma que los miembros de la familia deben tener una base fundamental de confianza y apego antes de poder aprender habilidades de comunicación y resolución de problemas. Esta teoría subraya la importancia de la existencia de un buen vínculo de apego como base para el aprendizaje de la resolución de problemas. Podríamos sospechar que los adolescentes que desafían de forma tan dolorosa y dramática a sus padres han sufrido de una forma más ostensible este daño en el vínculo de apego. Lo cual podría constituirse en un indicador de la necesidad de un abordaje terapéutico familiar más centrado en técnicas para lograr la reparación de dicho vínculo.

Por último, sólo hemos encontrado un autor (L. Cancrini) que se plantea, de forma teórica, la conveniencia de discriminar los tipos de abordaje. Concretamente, y en su tipología sobre las toxicomanías juveniles, este autor sugiere que los problemas de la esfera neurótica (toxicomanía B) pueden beneficiarse más de intervenciones de corte estructural, en tanto que los problemas de adicción vinculados con síntomas de la órbita borderline o francamente psicótica (toxicomanía C), se beneficiarían más de abordajes paradojales (para profundizar sobre su conceptualización, véase el capítulo n 6 de "La psicoterapia: gramática y sintaxis"). Ante los resultados que hemos obtenido en nuestra muestra, pensamos que igualmente podría ser aplicable una indicación diferenciada para los distintos tipos de problemas presentados, pero teniendo en cuenta, al menos en un plano hipotético, que hay familias donde el trastorno de comportamiento presentado por el chico puede obedecer a una dificultad de la organización familiar para aceptar los nuevos cambios que propicia el crecimiento (los padres estarían más ocupados en sus desacuerdos parentales o conyugales que en sus hijos) y, en otros casos, dicho trastorno tendría su base en las dificultades emocionales de los padres en sus respectivos procesos de desvinculación con respecto a sus familias de origen (estarían más ocupados en si mismos que en sus hijos). En la primera de las situaciones, donde serían más evidentes los problemas de disfunción estructural, la indicación terapéutica debería contemplar la reparación de la estructura; y en el segundo de los casos, donde quizás esté más presente el problema de la ruptura del apego, haya que trabajar en una doble vertiente: la reparación del vínculo padres-hijos y la resolución de las dificultades de desvinculación de los padres con relación a sus familias de origen. Nuevamente y a este respecto Cancrini afirma que "la circulación de mensajes y emociones no conscientes entre los padres y el hijo debe considerarse en suma como el elemento fundante de la psicopatología del joven". 


\section{9.- BIBLIOGRAFíA}

1. - Fishman H. Ch., Tratamiento de adolescentes con problemas, Barcelona, Ed. Paidós, $1^{\text {a }}$ Reimpresión, 1995

2. - Haley J., Trastornos de la emancipación juvenil y terapia familiar, Buenos Aires, Ed. Amorrortu, 1985.

3. - Knobel M., El Síndrome de la Adolescencia Normal, en Aberastury A. Y Knobel M.: La adolescencia normal. Un enfoque psicoanalítico, México, Ed. Paidós, 1998

4.- Simon F.B., Stierlin H., Wynne L.C., Vocabulario de Terapia Familiar, Barcelona, Ed. Gedisa, 1993.

5. - Waddell, M. Comprendiendo a tu hijo de 12-14 años, Barcelona, Ed. Paidós,1998.

6. - Cancrini, L, La psicoterapia; gramática y sintaxis. Manual para la enseñanza de la psicoterapia, Barcelona, Ed. Paidós, 1ª Edición,1991.

7.- Kobak R., Cole H., Ferenz-Gilles R., Fleming W., "Attachment and emotion regulation during mother-teen problem-solving: Acontrol theory analysis", Child Development, 1993, 64: 231 245

8.- Youniss J, Smollar J., Adolescent relations with mothers, fathers and friends, Chicago: University of Chicago Press, 1985.

9.- Montemayor, R., «Family variations in parent-adolescent storm and stress", Journal of Adolescent Research, 1986,1: 15-31.

10. - Boszormenyi-Nagy I., Intensive Family Therapy as process, en I. Boszormenyi-Nagy y J. L. Framo (comps.), Intensive Family Therapy: Theoretical and practical aspects, New York, Harper and Row, 1965

11.- Wynne L.C., «Un modelo epigenético de procesos familiares», en Falicov, C.J. (Compiladora): Transiciones de la familia. Continuidad y cambio en el ciclo de vida, Buenos Aires, Ed. Amorrortu, 1991.

12.- Olson D.H. (1991): «Tipos de familia, estrés familiar y satisfacción con la familia: una perspectiva del desarrollo familiar», En Falicov, C.J. (Compiladora): Transiciones de la familia. Continuidad y cambio en el ciclo de vida. Buenos Aires. Ed. Amorrortu. 1991

13. - Minuchin, S., Fishman H. Ch., Técnicas de Terapia Familiar, Barcelona, Ed. Paidós, $1^{\text {a }}$ Edición, 1984.

14. - Bowen M., De la familia el individuo. La diferenciación del símismo en el sistema familiar, Barcelona, Ed. Paidós, 1991.

15. - Matorin S., Greenberg L., "Family Therapy in the Treatment of adolescents", Hosp. Community Psychiatry, 1992, Jun; 43(6), 625-9.

16. - Villeneuve C., Roux N., "Family Therapy and some personality disorders in adolescence", Adolesc. Psychiatry 1.995; 20: 365-80

17. - Shapiro E.R., "The holding environment and family therapy with acting out adolescents", Int. J. Psychoanal Psychother, 1982-83;9: 209-26

18.- Szapocznik J., Río A., Murray E., Cohen R., Scopetta M., Rivas-Vázquez A., Hervis O, Posada V., Kurtines W., "Structural family versus psychodynamic child therapy for problematic Hispanic boys", J. Consult Clin Psychol, 1989, Oct;57(5):571-8

19. - Breunlin D.C., Breunlin C., Kearns D.L., Russell W.P., "Arevíew of the literature on family therapy with adolescents 1979-1987”, J. Adolesc., 1988, Dec; 1 1(4):309-34.

20. - Sourander A., Piha J., "A short-term, family oriented, child and adolescent psychiatric inpatient treatment program", Fam. Process 1996, Mar; 35(1):103-11 
ORIGINALES Y REVISIONES

21. - Abelsohn D., Saayman G.S., "Adolescent adjustment to parental divorce: an investigation from the perspective of basic dimensions of structural family therapy theory", Fam Process, 1991, Jun; 30(2):177-91.

22. - Brestan E.V., Eyberg S.M., "Effective psychosocial treatments of conduct-disordered children and adolescents: 29 years, 82 studies and 5.272 kids", J. Clin. Child. Psychol, 1998, jun; 27(2): 180-9.

23. - Kazdin A.E., "Practitioner review: psychosocial treatments for conduct disorder in children", J. Child. Psychol Psychiatry, 1997, Feb; 38(2):161-78.

24.- Mann S.J., Borduin C.M., Henggeler S.W., Blaske D.M., "An investigation of systemic conceptuaíizations of parent-chiíd coalitions and symptom change", J. Consult. Clin. Psychol, 1990 Jun; 58(3):336-44.

25. - Combrinck-Graham L., "Developments in family systems theory and research", J. Am. Acad. Child Adolesc. Psychiatry, 1990, Jul; 29(4):501-12.

26.- Gartland H.J., Day H.D., "Parental conflict and male adolescent problem behavior", J. Genet, Psychol, 1992, Jun; 153(2):201-9

27.- Smets A.C., Hartup W.W., "Systems and Symptoms: family cohesion/adaptability and childhood behavior problems", J. Abnorm Child Psychol,1.988, Apr; 16(2):233-46

28.- Diamond G.S.; Liddle H.A., "Transforming Negative Parent-Adolescent Interaccions in Family Therapy: From Impasse to Dialogue”, Fam. Proces, 1999, Vol. 38, n¹, 5- 26.

29.- Frielander, M.L. Wildman, J., Heaterington, L, Skowron, E.A., "What we do and don't know about the process of family therapy" Joumal of Family Psychology, 1994, 8: 390-416.

30.- Schmidt S.E., Liddle H.A., Dakof G.A., "Changes in parenting practices and adolescents drug abuse during multidimensional family therapy”, Journal of Family Psychology, 1996,10: 12-27.

31.- Young D.W., "Family factors in failure of psychotherapy", Am. J. Psychother., 1991, 45:4, 499-510.

32.- Firestone P., Witt, J.E., "Characteristics of families completing and prematurely discontinuing behavioral parent training”, Joumal of Pediatric Psychology, 1982, 7: 209-222.

33.- Robbins M.S.,Alexander, J.P., Newell, R.M., Turner, C.W. "The immediate effect of refraining on client attitude in family therapy", Journal of Family Psychology, 1996, 10: 28-34.

* Médico y Terapeuta Familiar

**Psicóloga y Terapeuta Familiar

Centro Psicológico y de Estudio de Familia

Correspondencia: Sebastián Girón García

Urbanización Los Gallos, 160-B

11130-CHICLANA DE LA FRONTERA (Cádiz)

Teléf. 956405136 - e-mail:giron.garcia@comcadiz.es

Fecha de recepción: 22-X-99 